\title{
Publicidad y cambio alimentario. Estudio de las razones de compra utilizadas en la publicidad de alimentos española
}

\section{Advertising and changes in eating habits. A study of the buying reasons used in Spanish food advertising}

\begin{abstract}
Juan Rey. Universidad de Sevilla
Recibido: 17-XI-2011 - Aceptado: 29-III-2012

Resumen:

El objetivo de este trabajo es el estudio de la vinculación entre las razones de compra utilizadas por los productos de alimentación y la transformación de los hábitos alimentarios. Tras aplicar un análisis de contenido a un corpus tomado de la prensa española del siglo XX, se obtiene el siguiente repertorio de razones de compra: autenticidad, belleza, bondad, ciencia, ecología, economía, envase, estatus, facilidad, novedad, salud, tradición y variedad. Lo más destacable es la frecuencia de aparición, la intensidad y la combinatoria de cada una de ellas. Todo lo cual permite observar la modificación de las preferencias alimentarias de los españoles.
\end{abstract}

Palabras clave:

Publicidad de alimentos, razón de compra, cambio alimentario, argumentación.

Abstract:

The aim of this article is the study of the connection between the buying reasons used to market food products and the transformation in alimentary habits. The following repertoire of buying reasons is obtained by applying a content analysis to a body of research collected from the Spanish press of the 20th century: authenticity, beauty, kindness, science, ecology, economy, packaging, status, ease, innovation, health, tradition and variety. The most outstanding aspect is the frequent appearance of these buying reasons, their intensity and their combination. All this enables to observe the modification of alimentary preferences in Spanish population.

Keywords:

Food Advertising, Buying Reason, Changes in Eating Habits, Argumentation. 


\section{Introducción}

En el ámbito universitario español los estudios sobre la alimentación, en general, han suscitado escaso interés, a diferencia de lo que sucede en otros países, como los anglosajones, en los que existe una larga tradición de análisis, desde las más diversas disciplinas, de las diferentes funciones de la comida. Esta desatención explica en cierto modo que, en el contexto de la publicidad, sean pocas las publicaciones sobre las razones de compra argüidas por los anunciantes de alimentación. No obstante, existen algunos estudios, aunque de carácter parcial. José Antonio Díaz Rojo, en "Lenguaje y reclamos de salud en la publicidad de los alimentos" (2003), analiza las razones vinculadas a aspectos médicos y sanitarios de la alimentación. Un planteamiento similar desarrolla en "El sanismo lingüístico. Recursos retóricos en la publicidad y etiquetado de alimentos" (Díaz Rojo et al., 2005). Y de manera más amplia y en colaboración con Ricard Morant i Marco y Debra W. Pixton, en "El culto a la salud y la belleza: la retórica del bienestar" (2006). En la misma línea se encuentra la investigación de Mabel Gracia Arnaiz: “Comer bien, comer mal. La medicalización del comportamiento alimentario” (2007). Un planteamiento más generalista y divulgativo ofrece la obra de Pilar Capanaga: "Salsa probiótica. La lengua de la publicidad alimentaria" (2003).

Sin embargo, la publicidad de alimentos no recurre a una sola razón -la salud-, sino a una variada gama de razones. Y ello se debe a que, en la sociedad de consumo, la alimentación ha traspasado el carácter funcional para adquirir nuevos valores y significados, ya que la alimentación se ha convertido en "un fenómeno complejo y multidimensional" en el que intervienen factores de diversa índole (Contreras Hernández y Gracia Arnaiz, 2008: 155). La función que la comida desempeña en la sociedad actual, sus implicaciones comportamentales y sus imbricaciones ideológicas han desembocado en la denominada "modernidad alimentaria", entendiendo por tal "los cambios alimentarios [habidos] en las sociedades industrializadas y posindustrializadas” (Díaz Méndez y Gómez Benito, 2008: 247). La novedad de esta investigación radica en relacionar, de una parte, las razones de compra de la publicidad de alimentos y, de otra, la modificación de los hábitos alimentarios, pues, como afirman Díaz Méndez y González Álvarez, la publicidad de alimentos es útil para analizar la sociedad de consumo porque "recrea [...] el contexto de consumo y los significados culturalmente relevantes para el consumidor" (2008: 106). Es decir, "la publicidad es un elemento importante para comprender cuáles son las tendencias sociales [y] qué imaginarios e identidades construye" (Marín Murillo et al., 2010: 36).

La presente investigación aborda el estudio de las razones de compra que han esgrimido los anunciantes de alimentación españoles y que han aparecido en la publicidad en prensa a lo largo del siglo XX. La hipótesis de partida es la siguiente: el establecimiento del catálogo de las razones de compra empleadas en la publicidad de alimentos y el análisis de su evolución permite registrar los cambios alimentarios ocurridos en España. Esta hipótesis se fundamenta, de una parte, en la relación existente entre "los valores, preferencias y expectativas que muestra la publicidad alimentaria" y "los cambios 
que los analistas expertos en sociología del consumo describen como propios del cambio en los consumidores" (Díaz Méndez y González Álvarez, 2008: 106); y de otra, en la aseveración de Perelman según la cual es posible "caracterizar las sociedades, no solo por los valores particulares que obtienen su preferencia, sino también por la intensidad de la adhesión que le conceden a tal o cual" (1994: 147). Todo ello permite, en primer lugar, establecer el repertorio de las razones de compra y su evolución a lo largo del siglo; y en segundo, observar, a partir de su evolución la transformación de los hábitos alimentarios de los españoles.

\section{Metodología}

El corpus está constituido por una muestra de anuncios de alimentación aparecidos en la prensa española entre 1900 y 2005. Se han elegido estas fechas porque delimitan un periodo lo suficientemente amplio para poder observar la evolución de la publicidad de alimentación. A fin de constituir un muestrario que se ajuste a la naturaleza de los productos analizados, se han elegido publicaciones generalistas (Abc, Blanco y Negro, Cambio 16, Diario 16, El Correo de Andalucía, El Liberal, El Mundo, El Noticiero Sevillano, El País, El Porvenir, La Unión y Pueblo) y revistas femeninas (Cosmopolitan, Diez Minutos, Elle, Época, Interviú, Hola, Marie Claire, Pronto, Semana, Telva, Tiempo y Vogue). Esta doble selección obedece a la evolución de la publicidad en prensa, pues, si hasta bien mediado el siglo la publicidad, en general, y de alimentación, en particular, aparece en los diarios, porque las revistas son escasas, en la segunda mitad, la publicidad de alimentación, sin desaparecer de los periódicos, se refugia en las revistas femeninas, que irrumpen masivamente en el mercado. De ahí que las publicaciones elegidas de los dos primeros tercios sean de carácter generalista mientras que, entre las del último tercio, figuren numerosas publicaciones destinadas a un público femenino.

Se ha realizado un muestreo sistemático. Los ciento cinco años se han dividido en lustros y de cada uno se han seleccionado treinta anuncios, que son el resultado de tomar seis ejemplos por año, que, a su vez, son el producto de elegir un anuncio cada dos meses. De esta forma se cubre todo el año y se atiende, además, a la estacionalidad del consumo de determinados productos. En cuanto a la selección de los anuncios, se ha atendido a un doble criterio: cantidad e información: se han seleccionado anuncios que presentan un componente redaccional extenso y, de entre ellos, se han elegidos aquéllos en cuyo texto figuran razones de compra.

Dado que son veintidós lustros, resulta un total de seiscientos sesenta inserciones, volumen que proporciona una idea acerca de las diversas razones de compra que aparecen a lo largo del siglo, ya que la organización de "una base de datos objetiva para el estudio de las relaciones que el lenguaje publicitario mantiene con los diferentes planos de la estructura social” permite, en palabras de Sánchez Guzmán, "dar cuenta de la evolución de las tendencias, actitudes y elementos transmitidos por la publicidad” (1985: 202). Por consiguiente, uno de los objetivos del análisis de contenido de la publicidad es estudiar la evolución diacrónica de los anuncios. Y esto es lo que se ha pretendido con este elenco: constituir un 
muestrario extenso y, sobre todo, homogéneo, a partir del cual obtener datos válidos acerca de la evolución de las razones de compra en el contexto de la sociedad española.

En cuanto al estudio de cada inserción en concreto, se ha analizado el componente redaccional. La elección del texto se debe a la extensión del periodo estudiado. La imagen tiene una gran preponderancia en la segunda mitad de siglo y sobre todo a partir del último tercio, sin embargo su presencia es escasa o nula en la primera mitad, especialmente en las primeras décadas. En cambio, el componente redaccional, si bien en los últimos decenios ha cedido en favor de la imagen, se mantiene más o menos uniforme a lo largo del siglo. A ello hay que sumar el hecho de que el elemento redaccional es el que vehicula información de una manera más precisa, frente a la mayor indefinición de la imagen, que, al margen de que proporcione información, necesita, a veces, el anclaje del texto para ser entendida correctamente. Asimismo no puede olvidarse que, en la tradición metodológica del análisis de contenido, el componente textual es el más estudiado frecuentemente. En este sentido, cada texto será sometido a un análisis de contenido siguiendo los postulados clásicos (Krippendorff, 2004).

Dada la naturaleza conceptual de las "razones de compra”, las unidades de registro del análisis de contenido serán de naturaleza temática. Así, por ejemplo, el tema "economía” se entenderá como la razón de compra del anuncio. El análisis de cada una de las inserciones proporcionará el catálogo de las razones de compra utilizadas y, al mismo tiempo, facilitará la frecuencia de cada una de ellas y su relación con las demás. Esto último es de suma importancia porque permitirá determinar cuáles son las razones de compra principales, cuáles las secundarias, de qué modo se combinan unas con otras y cuáles son las combinaciones más frecuentes, pues no puede olvidarse que en los textos publicitarios -al igual que en otros textos argumentativos (Adam y Bonhomme, 2000; Rey, 2010)- las razones de compra (argumentos de venta) no suelen figurar aisladamente, sino que aparecen constituyendo grupos, de modo que, entre todas ellas, se establece una interconexión que las vincula y relaciona hasta conformar un entramado argumentativo que es, en definitiva, el que persuade al receptor.

\section{Resultados}

Una vez realizado el análisis de las inserciones seleccionadas, el repertorio de los argumentos de ventas obtenido es el siguiente:

- Autenticidad. Se refiere a la originalidad del producto y se resume en "Rechace imitaciones".

- $\quad$ Belleza. Se refiere a la correlación existente entre el consumo del producto y la obtención de un cuerpo hermoso.

- $\quad$ Bondad. Se refiere a las excelentes características del producto. 
- $\quad$ Ciencia. Se refiere a los aspectos científicos del producto.

- $\quad$ Ecología. Se refiere a la protección del medio ambiente que desarrolla el producto, ya por su elaboración, por sus ingredientes o por su envase.

- $\quad$ Economía. Se refiere al precio del producto.

- $\quad$ Envase. Se refiere a los aspectos funcionales del recipiente en el que se presenta el producto.

- $\quad$ Estatus. Se refiere a la relación entre el producto y un grupo social determinado.

- $\quad$ Facilidad. Se refiere a la comodidad con que se prepara o consume el producto.

- $\quad$ Novedad. Se refiere a los aspectos innovadores del producto.

- Salud. Se refiere a los aspectos benéficos del producto y su incidencia en la salud del consumidor.

- $\quad$ Tradición. Se refiere al pasado como garantía del producto.

- $\quad$ Variedad. Se refiere a la diversidad que presenta el producto.

El análisis realizado ha proporcionado, además, información acerca de dos factores que resultan fundamentales a la hora de estudiar la evolución de las razones de compra: la frecuencia y el tiempo. Mediante el primero se determina la importancia de cada una de ellas; y mediante el segundo, su evolución cronológica.

\subsection{La frecuencia}

No todas las razones de compra aparecen con idéntica intensidad. A continuación se analizan los resultados obtenidos desde una doble perspectiva: desde su presencia en términos absolutos, y desde su presencia en solitario.

\subsubsection{Por presencia absoluta}

Como puede observarse en la tabla I, la prelación de las razones de compra en función de su presencia en el discurso en términos absolutos es la siguiente: bondad, salud, ciencia, variedad, tradición, novedad, envase, autenticidad, belleza, estatus, economía, facilidad y ecología.

\subsubsection{Por presencia en solitario}

Así como no todas las razones de compra tienen idéntica frecuencia, tampoco todas aparecen en el anuncio de la misma manera. Hay razones que aparecen en solitario y, en cambio, otras raramente o nunca se presentan solas sino que figuran como acompañantes. Lo más frecuente es que aparezcan constituyendo dúos o tríos. 
Tomando como referencia el número de veces que una razón de compra aparece en solitario, sin otras que la complementen, la prelación es la siguiente: estatus, envase, bondad, variedad, tradición, facilidad, autenticidad, salud, novedad, ciencia, belleza, ecología y economía (ver tabla II).

El estatus es la razón de compra que más veces figura en solitario $(50,28 \%)$. El hecho de que aparezca sola indica que -aunque se combine con la bondad $(22,21 \%)$, la variedad $(16,66 \%)$ y la tradición $(5,52 \%)$ - es una razón de compra poderosa.

El envase aparece como razón de compra exclusiva el 36,64\%. En las ocasiones restantes se combina, en mayor medida, con la novedad (23,38\%) y la bondad (20,16\%) y, en menor medida, con la salud, la tradición, la autenticidad y la variedad.

La bondad figura en solitario el 33,33\%. Aparece formando combinación con la salud (20,28\%), la variedad (12,91\%) y la tradición $(9,26 \%)$. Y, con unos porcentajes muy inferiores, con la ciencia, el envase, la belleza, la novedad, la autenticidad, el estatus, la economía y la facilidad.

La variedad aparece en solitario el 32,45\%. Lo más destacable es que su combinación con la bondad es de un 38,37\%. Con el estatus, la tradición, la ciencia, el envase y la salud se combina en unos porcentajes muy reducidos.

Igualmente sucede con la tradición, que en solitario representa el 29,62\% y combinada con la bondad, el 37,16\%. En mucha menor medida, se combina con la variedad, el envase, la salud, la autenticidad y el estatus.

La facilidad aparece en solitario el 20,34\% y, combinada con la bondad, el 39,10\%. Con la economía y la salud se combina en porcentajes muy inferiores.

Algo similar le ocurre a la autenticidad, que en solitario representa 19,16\%; combinada con la salud, el 35,39\%; y con la bondad, el 19,16\%. Con la autenticidad, el envase y la tradición se combina en porcentajes muy exiguos.

Un caso parecido presenta la salud, cuyo porcentaje en solitario es del 15,33\%, pero en combinación con la ciencia es del 33,49\%; con la bondad, del 20,91\%; y con la belleza, del 14,92\%. En mucha menor medida con la autenticidad, la novedad, la economía, la tradición, la variedad, el envase, el estatus y la facilidad.

La novedad es raro que aparezca en solitario (6,66\%). Pero es frecuente que aparezca combinada con el envase $(23,33 \%)$, la salud (23,33\%), la bondad (16,66\%) y la ciencia (13,33\%). Y en menor medida con la tradición, la variedad y la belleza.

A la ciencia le sucede otro tanto, que es infrecuente su aparición en solitario $(4,48 \%)$, pero muy frecuente que aparezca en combinación con la salud $(70,10 \%)$, la bondad $(13,37 \%)$ y la novedad $(12,81 \%)$.

En última instancia figuran la belleza, la ecología y la economía, que nunca aparecen como razones de compra únicas. 
Si se tienen en cuenta la frecuencia de aparición en términos absolutos y la frecuencia de combinación con otros argumentos, todo ello permite organizar las razones de compra en función de su autonomía, entendiendo por ésta la mayor o menor capacidad que tiene una razón de proporcionar un argumento lo suficiente persuasivo para que no sea necesaria la copresencia de otras. Puede hablarse, pues, de razones de compra mayores y menores. Aquéllas son fuertes, independientes. Éstas, débiles, dependientes. Aquéllas presentan un carácter autónomo. Éstas, complementario.

Una vez realizada la media entre la presencia absoluta y la presencia en solitario, las razones de compra se distribuyen en una escala de autonomía que, de más a menos, es la siguiente: bondad, estatus, envase, salud, variedad, tradición, autenticidad, facilidad, ciencia, novedad, belleza, economía y ecología. Esta escala permite, a su vez, la distribución de las razones en tres bloques: razones mayores (bondad, estatus, envase, salud y variedad), razones menores (tradición, autenticidad, facilidad, ciencia y novedad) y proto-razones (belleza, economía y ecología).

\subsection{El tiempo}

Desde el punto de vista de su presencia a lo largo del siglo, no todas las razones de compra ofrecen idéntico desarrollo. Como puede observarse en la tabla III, hay razones que recorren toda la centuria y otras que son de reciente aparición. No obstante, y como se verá con más detalle, hay notables diferencias entre sus diversas trayectorias.

\section{Discusión}

Los datos obtenidos muestran la evolución de las razones de compra utilizadas por los anunciantes españoles y, al mismo tiempo, proporcionan información acerca de las transformaciones habidas en el ámbito de la alimentación en España, ya que, además de los aspectos cualitativos, también se han tenido en cuenta los años en los que las razones de compra aparecen o desaparecen, fechas que indican el momento en el que los consumidores les otorgan o retiran su favor.

\subsection{Razones mayores}

Las razones mayores son las que funcionan o pueden funcionar de manera autónoma e independiente en el discurso publicitario y que, por tanto, no necesitan la concurrencia de otras para promocionar un producto, porque son lo suficientemente fuertes para persuadir por sí solas. Son: la bondad, el estatus, el envase, la salud y la variedad.

\subsubsection{La bondad}

Si hay una razón de compra que recorra el siglo con similar intensidad ésa es la bondad. No en vano la publicidad promociona productos y servicios, y su función básica es cantar sus excelencias. Si se tiene en cuenta que la bondad, tanto por orden decreciente como por bloques de frecuencia, ocupa el primer lugar y el segundo por combinatoria, no cabe la menor 
duda de que se trata de una razón de compra fuerte, autónoma, independiente, poderosa y frecuente. Es decir, la bondad es la razón de compra por antonomasia, al margen de épocas y modas.

No obstante, esta magnitud y uniformidad no son suficientes para comprender esta razón de compra en su toda amplitud si no se realizan dos observaciones previas. Primera, que la bondad no se conjuga de idéntica manera con otras razones. Se combina, en mayor medida, con la salud, la variedad y la tradición, y, en menor, con la ciencia, el envase, la belleza, la novedad, la autenticidad, el estatus, la economía y la facilidad. Y segunda, derivada de la anterior, que el significado de bondad varía a lo largo del siglo.

La bondad, en función de su vinculación con unas u otras razones de compra, manifiesta tres significados básicos, significados que, al hacer su aparición, no eliminan el anterior sino que conviven en mayor o menor medida, y que sirven para establecer otras tantas etapas. En un primer momento, la bondad se vincula a lo sabroso; en un segundo, a lo saludable pero no-sabroso; y en un tercero, a lo saludable y sabroso. La primera etapa nace con la publicidad, la siguiente se desarrolla a partir del último tercio del siglo pasado, y la última aparece en el tránsito del siglo XX al XXI. Y es en estas dos últimas cuando la bondad se vincula con la salud y la ciencia, e indirectamente con la belleza.

El significado básico de la bondad se fundamenta en las características tradicionales de la alimentación: si es sabroso (en el sentido de exquisito, delicioso y rico), es bueno. Y éstas son las cualidades que, con intensidad variable, explota la publicidad desde su nacimiento hasta la actualidad, si bien su época dorada se sitúa en los cuartos centrales del siglo XX. La bondad se expresa mediante nociones referidas a lo sabroso, exquisito, delicioso y rico del producto:

- Sabroso: "Es la base de la cocina sana y sabrosa" (extracto de carne Liebig, 1910ㄹ), "Es la manera más natural de hacer sabrosas salsas" (tomate triturado Tomator, 1990).

- $\quad$ Exquisito: “La exquisita galleta para desayuno, merienda y postre" (Artiach, 1920), "La exquisitez se reconoce” (yogur Danone, 2000).

- Delicioso: "Pruebe los deliciosos chocolates de los RR PP Benedictinos" (1920), "Una deliciosa novedad” (queso Bonsi, 2000).

- Rico: “Es el desayuno más rico” (chocolate Jaime Boix, 1900),"Y a las amas de casa, nuestro reconocimiento por los mimos que dieron a sus hijos al poner en la leche el alimento rico, rico” (cacao Gua Cao, 1970).

Esta primera etapa queda sintetizada en el anuncio de La Estrella: "Cuerpo. Aroma. Sabor. Textura. Son palabras que definen un buen café" (1970). Es decir, son conceptos que sirven para establecer la bondad de un producto. Cuando en la

1 La fecha entre paréntesis después del texto publicitario no hace referencia al año en que aparece el anuncio sino a la década. 
misma década Danone proclame que su yogur es “fresco, natural, puro, sano y nutritivo", ya está apuntando la segunda fase, porque a las características tradicionales se le han sumado otras nuevas: natural, puro, sano. En la segunda etapa, la bondad del producto se vincula con su carácter saludable. Ya no importa que sea delicioso o esté exquisito, lo que ahora interesa es que sea beneficioso para la salud. "Lo bueno de comer sano" dice el eslogan de El Pozo en las últimas décadas del siglo.

Esta vinculación bondad-salud nace al amparo del proceso de medicalización que sufren los alimentos a partir de los años ochenta. Y este mismo proceso es el que explica que la salud sea la razón de compra que más veces se conjugue con la bondad (v. apdo. 4.1.4 ${ }^{2}$. Ello se debe a que en todas las sociedades, pero especialmente en la de consumo, se establece una estrecha conexión entre alimentación y salud (Fazio, 2008: 28; Vallone, 2009: 99). Así lo manifiesta la publicidad española, aunque con una intensidad variable a lo largo del siglo. A comienzos del siglo XX aparecen algunos anuncios en los que se vinculan ambas razones: "Todos esperan con ansiedad el momento de paladear las exquisiteces de los muy nutritivos chocolates de los RRPP Benedictinos" (1910), anuncio en el que se aúna bondad ("exquisiteces") y salud ("nutritivos"). No obstante esta combinación se incrementa a partir de los años ochenta, justo en el momento en el que se intensifican las corrientes sanistas que, unidas al hedonismo imperante y la importancia alcanzada por la imagen personal, "imponen [hacia fin de siglo] una alimentación adecuada y, sobre todo, sana" (Oliva et al., 2008: 507).

La publicidad se hace eco de estas nuevas razones de compra y las utiliza como reclamos, ya que el consumidor ha cambiado sus gustos y tendencias en alimentación durante el último tercio de siglo pasado, como expone Mabel Gracia Arnaiz en su excelente estudio "La transformación de la cultura alimentaria. Cambios permanentes en un contexto urbano" (Barcelona, 1960-1990) (1997). El eslogan de Pascual sintetiza el espíritu de la época: “La calidad y tu salud, nuestra razón de ser” (2000).

Ahora los aspectos sabrosos del producto pasan a ocupar un lugar secundario, mientras que son los salutíferos los que ocupan el primer plano, porque el alimento se ha convertido casi en un fármaco. El binomio bondad-salud suele llevar aparejada la presencia de otras razones, que frecuentemente son la belleza y la ciencia. La primera, como consecuencia del consumo de un producto saludable, y la segunda, unas veces, como causa y, otras, como garante de la bondad y saludabilidad del producto. Resulta interesante observar que estas triples combinaciones aparecen a finales de siglo XX. La primera en sumarse es la belleza, en los años setenta y ochenta, y luego la ciencia, en los noventa, justo en el momento en el que, parafraseando a Perelman, se intensifica "la adhesión" de los consumidores a estos conceptos, promovidos por las corrientes hedonistas y sanistas. De este modo viene a establecerse una indisoluble ecuación entre salud y belleza, que se fundamenta en la saludabilidad del producto que, a su vez, deriva del proceso de "medicalización" al que ha sido sometido a en las últimas décadas la alimentación (Gracia Arnaiz, 2007: 238). Así puede observarse en: "Queso bajo en calorías, pero con sabor. Bonsi, el queso a tu medida" (1980) (v.apdo.4.3.1).

Dado que las razones de compra suelen aparecer en combinación con otras, cuando sea necesario, se hará referencia a otros apartados a fin de proporcionar mayor información sobre cada razón. 
Este proceso de medicalización -surgido de las corrientes sanistas y del afán de tener un cuerpo modélico- es el que ha determinado que la ciencia se combine con la salud y la bondad. Son numerosos los productos de alimentación que recurren a la ciencia como reclamo: "cereales hidrolizados" (papillas Nestlé, 1990), "Péptidos lácteos" (margarina Flora Pro-activ, 1995), “ácidos grasos omega 3 y DHA” (leche Puleva, 2000) (v.apdo.4.2.4). La ciencia se convierte en el fundamento de la bondad del producto, de su carácter salutífero y de sus efectos estéticos.

Es tanta la terminología científica que figura en la publicidad de alimentos que el cuerpo de texto termina convirtiéndose en el prospecto de un fármaco. En los medicamentos lo importante no es el sabor, sino su incidencia en la salud. Y así lo manifiestan los siguientes anuncios: “Una cucharada de mayonesa te aporta vitaminas E antioxidante, omega 3 y 6" (genérico de mayonesa, 1990), “Tu combustible. Con fósforo, magnesio y calcio” (yogur Yosport, 2000). Los alimentos funcionales son la máxima manifestación de esta tendencia (apdo. 4.2.4).

Todo ello posibilita el paso a la tercera fase, que se da en el tránsito del siglo XX al XXI y representa la solución de la "paradoja alimentaria". Si en la segunda fase lo saludable estaba reñido con lo sabroso, en ésta lo saludable puede ser sabroso. La obsesión por los aspectos médicos de la comida no ha hecho desaparecer del todo sus aspectos placenteros, de ahí que “cada vez más se demanden alimentos funcionales con los atributos sensoriales de los tradicionales” (Juárez, 2007, 31). Es decir, unas magdalenas fabricadas con todos los ingredientes químicos que posibilitan los avances científicos en el ámbito de la bioalimentación (un medicamento en definitiva), pero con el olor, el sabor y la textura de las de la abuela. Así lo recogen los anuncios siguientes: "Quítate las grasas pero no renuncies al sabor de la leche” (Pascual desnatada, 1990), "Rebaja calorías sin rebajar sabor" (mayonesa Ybarra light, 1990). Incluso productos tan poco livianos como la mermelada resuelven la paradoja: "Mucho placer, pocas calorías" (Heros Diet, 2000). Antes de concluir cabe indicar que muchos de los aspectos analizados en la segunda y tercera etapa se complementan con otros que se desarrollan al estudiar la salud (v. apdo. 4.1.4), la ciencia (v. apdo. 4.2.4) y la belleza (v. apdo. 4.3.1).

La variedad es la segunda razón que porcentualmente se combina con la bondad. La excelencia del producto se asocia a la diversidad bajo la cual se presenta, a la pluralidad de formas, a la multiplicidad de funciones (v. apdo. 4.1.5). Si bien es un reclamo que se usa con moderación a lo largo del siglo, su empleo se intensifica a partir de los años sesenta y setenta, justo cuando se inicia, de una parte, la fragmentación del mercado durante el desarrollismo económico de los últimos años del franquismo y, de otra, su atomización con la implantación de la sociedad de consumo. La tercera razón que porcentualmente se conjuga con la bondad es la tradición (v. apdo. 4.2.1).

\subsubsection{El estatus}

El estatus es la razón de compra que atribuye al producto características de singularidad, exclusividad y prestigio, y que, por tanto, solo está al alcance de una minoría. Se trata de una razón que recorre todo el siglo, desde los "panes de lujo" de 
la panadería San José (1900) hasta los bombones de Ferrero Rocher y sus fiestas mundanas (2000), sin embargo presenta matices diversos y diferente intensidad: baja en el primer tercio, moderada en el segundo y fuerte en el tercero. Aunque la sociedad de consumo ha dado lugar a la "democratización de la alimentación” (Martínez Álvarez, 2008: 9), aún se sigue utilizando el viejo recurso de la exclusividad alimentaria como argumento de compra. Se trata de un vestigio de la etapa preconsumista en la que el consumo de determinados productos era señal inconfundible de la posición social. El consumismo ha banalizado este argumento y, si bien lo usa para productos que en épocas pasadas tuvieron carácter elitista como el chocolate ("Proveedor de la Casa Real", Menier, 1920), también lo emplea para vender productos de consumo diario: queso en porciones (“Tiene más aceptación, más prestigio”, El Caserío, 1980), salchichas (“'Salchicha de Frankfurt!, Calidad y prestigio”, La Piara, 1980), queso curado (“Un lujo razonable”, Campo Real, 2000).

Con unos porcentajes considerables, el estatus se combina con la bondad ("Buitoni, el buen gusto italiano. Formas, imaginación, buen gusto. El gusto por la pasta", 1990) y la variedad ("Chocolate Elgorriaga, con su extenso surtido de especialidades, le ofrece una de las posibilidades de quedar bien en cada momento. Una caja de bombones, en cualquiera de sus tamaños, o una caja de Pralinectar, con treinta bloques de surtidos”, 1970).

\subsubsection{El envase}

El recurso al envase es moderno. Salvo algún caso aislado, esta razón de compra es de reciente aparición. No se remonta más allá de los años sesenta y es, en esta época, cuando aparece con profusión. A partir de esta década España inicia su carrera hacia la sociedad de consumo (Montero et al., 2010) y la publicidad es la encargada de dar a conocer los nuevos productos, una de cuyas ventajas es ahora su presentación. Son numerosos los anuncios que acuden al envase como argumento de venta. Gracias a ellos pueden observarse las novedades técnicas ("El chorizo Revilla se comercializa envasado al vacío, permitiendo su óptima conservación durante dos meses”, 1970) y las nuevas tendencias sanitarias ("Máxima higiene. Totalmente hermético", flan Dhul, 1980), demográficas ("El nuevo Avecrem en sobrecitos individuales", 1990) y ecológicas (“Font Vella ha sido pionera en la utilización del plástico reciclado P-PET”, 2000). El envase se asocia con la novedad (v. apdo. 4.2.5):

Café Saimaza en su nuevo envase al vacío. Gracias a este nuevo envase, el buen café Saimaza conserva su frescor mucho más tiempo y, sobre todo, usted puede comprobar su aroma antes de comprarlo. El procedimiento de envasado "Fresco System" ha merecido distinciones entre las que destaca el Oscar Mundial del Embalaje "Word Star". Compruébelo: sólo tiene que presionar ligeramente la bolsa de café Saimaza para que, a través de sus válvulas Aromacti, perciba un poco de su delicioso aroma de café recién tostado (1970).

Y con la variedad (v. apdo. 4.1.5): 
“Desde ahora, no todas las tapas de La Piara son negras. Paté de pollo. Paté de jamón York. Paté de campagne. Paté de ave. Paté de sobrasada. Paté de chorizo. Desde ahora, no todas las tapas de La Piara son negras. Y son así para ayudarte a identificar estos 6 nuevos y deliciosos sabores de los patés La Piara" (1980).

\subsubsection{La salud}

La relación entre salud y publicidad de alimentos puede resumirse en un arco que comienza con un anuncio de La Lechera y concluye con otro de Danone. En el primero, una madre, orgullosa, muestra su hijo al espectador mientras el texto exclama: “iMiren qué hermoso! Así se crían los niños que toman leche condensada La Lechera. Désela a su niño, señora, verá qué gordito de pone” (1920). En el segundo, hombres y mujeres de cuerpos escultóricos nadan en la piscina de un balneario mientras el eslogan proclama: “Cuerpos danone” (1990). La salud como reclamo ha estado presente en la publicidad durante todo el siglo. No obstante, su planteamiento ha cambiado notablemente de una a otra época. Así lo expresan los dos anuncios recogidos. En uno, la salud se vincula con "gordito"; en otro, con delgadez. Lo que viene a subrayar que la salud es "un constructo conceptual y conductual modificable en el tiempo" (Davo y Álvarez-Dardet, 2003: 61). La salud es una razón de compra que recorre toda la centuria como un guadiana, ya asociado a la ciencia, ya a la bondad, ya a la belleza.

El binomio salud-ciencia recorre todo el siglo, pero con vinculaciones diferentes (v. apdo. 4.2.4). En las primeras décadas figura asociado a los reconstituyentes ("Un alimento perfecto para los enfermos como para los niños más delicados”, Revalenta du Barry, 1910), el agua mineral ("Preservativa de enfermedades e infecciones y estar libre de microbios", Vichy Catalán, 1920), la leche infantil ("No corráis ese riesgo con vuestros hijos y criadlos con Glaxo, el mejor alimento para criar niños, que está garantizado, libre de los gérmenes de la tuberculosis", 1930) y el chocolate ("Excelente compuesto de cacao, azúcar y leche constituye un poderoso alimento. Es el desayuno más rico, nutritivo y práctico”, Jaime Boix, 1900). Tras un paréntesis durante los años cuarenta-sesenta, en el que el tándem salud-ciencia atenúa su presencia, vuelve a reaparecer hacia los años setenta. Ahora presenta varias novedades: $a$ ) está asociado a casi todo tipo de producto, $b$ ) el carácter científico se intensifica a medida que avanza el siglo hasta transformarse en discurso médico, y $c$ ) se convierte en el camino indispensable para alcanzar un cuerpo canónico.

De estas características derivan las combinaciones más frecuentes: salud-bondad y salud-belleza. El hecho de que la salud aparezca vinculada a cualquier producto de alimentación determina que su bondad depende de su incidencia favorable sobre la salud. Es lo que se ha denominado la segunda fase de la bondad en la que lo bueno se identifica con lo saludable (v. apdo. 4.1.1). Son numerosos los ejemplos: “Una alimentación sana, equilibrada y práctica” (pan Bimbo, 1980), "Fontaneda: cereal, fibra y mucho sabor" (galletas, 2000). La obsesiva preocupación por la salud y la incidencia que la alimentación tiene sobre ella se ha exacerbado en la sociedad de consumo hasta tal punto que de la "medicalización de la sociedad" se ha pasado a la "medicalización de la publicidad" (Gracia Arnaiz, 2007: 238). La tradicional vinculación ali- 
mentación-salud ha superado el ámbito médico y se ha convertido en una razón de compra más. Ello se debe a que "del propósito más primario de satisfacer el hambre y mantener el organismo se pasa, al comenzar la segunda mitad del siglo $\mathrm{XX}$, al de suministrar al organismo los nutrientes necesarios y proporcionarle alimentos que no actúen como vectores de enfermedad" (Arpe Muñoz, 2008: 35). Ya no hay alimentación sino nutrición.

La publicidad, a instancias de la sociedad, intensifica la relación salud-ciencia y su discurso se trufa de vocablos científicos hasta "tener la impresión de entrar en un laboratorio" más que en una tienda (Gracia Arnaiz, 2003, 45). Y es en este contexto en el que hay que situar la relación salud-belleza, pues la salud no es el objetivo, sino el medio para lograr un cuerpo hermoso mediante la ayuda de una alimentación, más que sana, científica. De ahí que sea a partir de los años ochenta, una vez consolidado el discurso médico de la alimentación, cuando se procede a la exaltación del cuerpo y a la vinculación de salud y belleza, exaltación que se acentúa con el nuevo siglo. En el siguiente texto confluyen la bondad, la salud, la ciencia y la belleza:

Tienes que suspender el exceso de grasa, para tener una figura sobresaliente y para ganarle la carrera al colesterol. Porque el exceso de grasa en la alimentación no sólo engorda, sino que aumenta el colesterol y perjudica tu salud. Leche Pascual desnatada es tu mejor aliada. Tiene la nota máxima en proteínas, vitaminas, minerales, un cero en grasa y matrícula de honor en calidad. Leche Pascual desnatada ayuda a no engordar (1990).

\subsubsection{La variedad}

No es suficiente que el producto sea bueno, debe ser además adecuado. La implantación de la sociedad de consumo supone la fragmentación del mercado y, por ello, el mismo producto tiene que satisfacer la demanda de los diferentes grupos de consumidores respondiendo a sus necesidades personales, sociales, laborales... La variedad significa la fragmentación del propio producto, pero también la del tiempo y el lugar de su consumo. Se trata de una razón de compra que recorre todo el siglo, desde las variedades de la casa Matías López en 1900 ("Especialidad en bombones de chocolate con cremas finísimas, caramelos suizos, fondant y dulces varios”) hasta las mousses de Chamburcy en 1990 ("Voy a empezar por la mousse de chocolate. Seguiré con la mousse de limón y turrón. Y después la mousse de café”). Dado que la variedad está relacionada con la fragmentación del mercado, la presencia de esta razón de compra, si bien tiene una trayectoria discreta en el primer y segundo tercio del siglo, se acentúa en el último, en el cual no hay producto que no recurra a la variedad, como puede observarse en los ejemplos siguientes:

"La riquísima galleta para desayuno, merienda, postres y para todas las horas" (Artiach, 1930), "Chocolates Elgorriaga le recomienda su extensa gama de cajitas de bombones en los tamaños más variados” (1950), "Variedad en platos, ensaladas, entrantes, aperitivos. La mejor forma de variar es sacar algo de Albo de la despensa. Para variar" (1970). "El color rojo es para la leche entera con toda su grasa. El color azul se ha dado a la nueva leche, con la mitad de grasa. El color verde sigue siendo para la leche desnatada” (Asturiana, 2000). 
El mayor aliado de la variedad es la bondad. La variedad es porcentualmente la segunda razón de compra que se combina con la bondad y ésta es la primera en aliarse con la variedad (v. apdo.4.1.1). Bondad y variedad son dos razones que suelen figurar juntas: "Cien clases diferentes de galletas preparadas con alimentos puros y frescos, de calidad inmejorable" (Cuétara, 1970), "Cafés La Estrella, en grano, molido o soluble. Sólo así se llega a ser La Estrella del café” (1990).

\subsection{Razones menores}

Las razones de compra secundarias son las que presentan un carácter semiindependiente. Son argumentos que no actúan de forma autónoma. Dado que no son lo suficientemente fuertes para persuadir por sí solas, funcionan como complemento de las denominadas razones mayores. Se trata de razones de segundo orden, pero no por ello menos importantes a la hora de incitar a la compra, como se verá en los apartados siguientes, pues puede suceder que una razón principal (la bondad) es la vía para alcanzar una secundaria (la autenticidad). Las razones menores son: la tradición, la autenticidad, la facilidad, la ciencia y la novedad.

\subsubsection{La tradición}

La trayectoria de la tradición como razón de compra es similar a la de la variedad, pues su omnipresencia en el último tercio del siglo es parecida y su combinación con la bondad también. La tradición es una razón moderna. En épocas anteriores se ha usado a veces (“50 años de éxito”, papillas Nestlé, 1910; “Las delicias de nuestras abuelas”, chocolate de los RRPP Benedictinos, 1920), pero son casos poco representativos. Es a partir de los años sesenta cuando comienza a recurrirse a ella para certificar la bondad del producto, justo en un momento en el que la sociedad española está dejando de ser agrícola para transformarse en urbana. Paradójicamente, el éxodo del campo a la ciudad conlleva la exaltación de lo rural que, desde la perspectiva urbana, es percibido de manera distorsionada, pero elogiosa. Es en este momento cuando el mensaje publicitario se hace eco de la división entre lo moderno y lo tradicional, entre la ciudad y el pueblo. Y lo popular, con sus connotaciones de sencillez (frente a artificiosidad), durabilidad (frente a caducidad), pureza (frente a contaminación) y naturalidad (frente a industrial), se convierte en el garante de la bondad del producto. En este sentido, la mayonesa Kraft "está hecha con los mismos ingredientes naturales que usted emplearía para hacer la auténtica mayonesa" (1970), los cafés La Estrella son "el resultado de una larga tradición” (1980) y los sabores de las aceitunas La Española “están inspirados en la gastronomía mediterránea tradicional” (2000).

El recurso a la tradición coincide con la segunda y tercera fase de la bondad (v. apdo. 4.1.1). Esta razón de compra es uno de los integrantes del conglomerado ideológico que sirve para resolver las "paradojas" de la publicidad alimentaria: por una parte, se abandona el campo, pero se añora en el tráfago de la ciudad; y por otra, se pretenden alimentos que sean fárma- 
cos, pero con el sabor "de toda la vida”. La tradición viene a resolver estas contradicciones, ya que su función es traer el campo a la ciudad mediante el consumo de productos que tienen la impronta de lo popular, lo artesano, lo sencillo.

\subsubsection{La autenticidad}

A lo largo del siglo se observa, en muchos anuncios, la presencia de una advertencia relativa a la autenticidad del producto, lo que indica que las empresas se preocupan por defenderse de los imitadores. La razón de compra de la autenticidad recorre toda la centuria, si bien se acentúa al comienzo de siglo, unido a la salud, y al final, asociado a la bondad.

En un primer momento, la autenticidad figura en los anuncios de reconstituyentes: "Desconfiar de imitaciones y falsificaciones" (vino de peptona Chapoteaut, 1900), "Exíjase la lata en español con envoltura verde y el nombre de los importadores exclusivos" (esencia de pollo Brand, 1920). En menor medida, también figura en los productos de despensa: "Exíjase expresamente la marca La Lechera, la más acreditada en todo el mundo" (leche condensada, 1910), "Fijarse en el nombre Maggi y las etiquetas en rojo y amarillo" (1930). Tras una trayectoria discreta durante las décadas centrales del siglo, su presencia se acentúa a partir de los años sesenta, ahora para subrayar la bondad del producto: "Identifique el auténtico queso manchego. Todo lo demás son imitaciones. Adherida a esta etiqueta encontrará una contraetiqueta numerada expedida por el Consejo Regulador que garantiza el origen y la calidad del queso manchego" (1990), "Solicite siempre el certificado y la etiqueta correspondiente. Ternera Gallega, la carne con carné" (2000).

\subsubsection{La facilidad}

La razón de compra de la facilidad alude a la comodidad con que se prepara o consume el producto. Es una razón reciente. Aparece en los años sesenta. Igual que la variedad, la facilidad puede ser considerada una forma de adecuación, ya que la fácil preparación hace desaparecer o atenúa algunas de las circunstancias adversas del consumidor (inexperiencia, falta de tiempo...). La facilidad recorre la segunda mitad de siglo de manera creciente, desde la "preparación rápida y eficaz" del flan Bakol (1960) hasta el "sopinstant” de Avecrem (1990). Su gran aliada es la bondad, a la que, como razón secundaria, aparece supeditada: "Nesquik da a la leche este delicioso sabor a chocolate ¡que tanto les gusta a los niños! (cacao en polvo, 1970).

\subsubsection{La ciencia}

En reiteradas ocasiones, al hablar de otras razones de compra, se ha hecho referencia a la ciencia, lo que viene a indicar que se trata de una razón complementaria. El carácter científico es una especie de humus sobre el que se asienta la salud (y su derivado la belleza) y, en menor medida, la bondad. La vinculación ciencia-salud recorre todo el siglo, aunque con 
fluctuaciones. En las primeras décadas aparece asociada a los reconstituyentes (v. apdo. 4.1.4). Y es en esta época cuando se observa la presencia del "factor laboratorio", el "factor médico" y el "factor autenticidad" como subargumentos de la ciencia.

El primero consiste en recurrir a instituciones de investigación para justificar el carácter científico del producto y, por tanto, su excelencia: "Elaborados según la fórmula aprobada por los Laboratorios Químicos Municipales de Madrid, Pamplona y San Sebastián” (chocolate La Trapa, 1910). El segundo es el recurso a la terminología médica para subrayar la bondad del producto y la adecuación al problema tratado, lo que determina que el texto se llene de vocablos médicos (v. apdo. 4.1.4): "Curativa de los eczemas, herpes, seborreas, sarna, erisipela" (agua mineral Loeches, 1910). Y el tercero es el recurso a la autenticidad, que garantiza la originalidad y, por tanto, la bondad del producto. Y mediante el cual se advierte de las imitaciones: "Exigid sobre las etiquetas de los tarros la marca en tinta azul" (extracto de carne Liebig, 1910) (v. apdo.4.2.2).

Tras varias décadas de letargo, el argumento científico reaparece en los años sesenta, pero lo hace en un nuevo contexto, de modo que ahora la ciencia -apoyada en las concepciones higienistas de salud, sanistas de alimentación y somatocéntricas de estética (Díaz Rojo, 2001-2002: 112)- se vincula a la bondad del producto. De esta forma viene a establecerse una ecuación entre ciencia-salud-bondad cuyo resultado es un cuerpo bello. La vinculación ciencia-bondad aparece en el último tercio del siglo y es el resultado de la confluencia de dos nuevas corrientes: la "medicalización de la alimentación" (Gracia Arnaiz, 2007: 238) y la "aculturación del conocimiento científico” (Davo y Álvarez Dardet, 2003: 61). La primera consiste en la extensión de los postulados de la farmacopea a la alimentación, debido a que "la interpretación biomédica enfatiza los aspectos fisiológicos [de los alimentos] en detrimento de los sociales”. Ello hace que los ingredientes se transformen en nutrientes. La segunda consiste en la divulgación de las innovaciones médicas y su incorporación al conocimiento popular, lo que "provoca también cambios conductuales" en relación con la alimentación.

En este sentido, la bondad nunca figura sola junto a la ciencia, sino que siempre aparece formando parte del trío cienciabondad-salud, porque la bondad del producto, refrendada por la ciencia, incide en la salud. El siguiente anuncio es un excelente ejemplo de cuanto se ha dicho:

Nadie pone tanto en un brik como leche Pascual. 1) Selecto ganado vacuno. Nuestra leche procede de las mejores vacas, alimentadas con pastos naturales y controladas sanitariamente. 2) Ordeño automático. Sistema que evita los riesgos de contaminación provocados por el ordeño manual. 3) Tanque de frío. La leche pasa directamente desde la sala de ordeño a los tanques de frío. Se impide así el desarrollo bacteriano. 4) Cisternas laboratorios. Diariamente se recoge la leche con cisternas isotérmicas, auténticos laboratorios rodantes que analizan la leche en origen. 5). Control de recepción. Sólo se admite en nuestros centrales leche que supera los exhaustivos controles de calidad Pascual. 6) Uperización. El más moderno tratamiento para 
dar larga vida a la leche no superado por ninguna otra marca en el sector lácteo. 7) Envasado en brik. El brik impide la más mínima alteración de la leche por la acción de la luz y ofrece grandes ventajas. 8) Control final de calidad. Una vez envasada la leche, se efectúan rigurosos análisis que garantizan la máxima calidad (1990).

La última fase de este proceso lo representan los alimentos funcionales. La obsesiva preocupación por los aspectos salutíferos de los alimentos, alentada por la medicalización de la sociedad y la difusión de las novedades médicas, ha provocado que los productos, al amparo de la visión cientificista de la alimentación, se conviertan en medicamentos. Su bondad radica en su carácter farmacéutico. De ahí que algunos los llamen "alicamentos", "nutracéuticos", "alimentos terapéuticos", "alimentos de diseño" o "alimentos funcionales" (Murcia et al., 2008: 139). Así puede verse en: "Tu aliado contra el colesterol" (yogur Danone, 2000), "Flora ayuda a regular el colesterol al contener Omega 3 y 6. Tulipán te aporta energía y vitaminas A, D y E” (genérico de margarina, 2000). En última instancia, dado que estos conceptos, procesos y productos son nuevos, la ciencia suele llevar aparejada la novedad (v. apdo. 4.2.5).

\subsubsection{La novedad}

Si hay una razón de compra que en publicidad resulta intacta a pesar de su abuso, es la novedad. No obstante, no es una razón fuerte, pues son raras las ocasiones en las que figura en solitario. En cambio, es mucho más frecuente que aparezca como complemento de otras razones, preferentemente el envase, la salud, la bondad y la ciencia. En cuanto al tiempo, le sucede como a otros argumentos, que, aunque tiene apariciones fugaces a lo largo del siglo, su presencia se consolida en los años sesenta y desde entonces mantiene una trayectoria ascendente.

La novedad es un excelente complemento del envase, pues éste supuso una gran innovación en los años sesenta y siguientes (v. apdo. 4.1.3). El "frasco de cristal de cierre hermético" de Eko, la "nueva y cómoda mantequera" de Reny Picot, la "flanera higiénica" de Dhul, el método "Fresco System" con "válvula Aromatic" de Saimaza, el "brik cómodo e higiénico" de Tomator, los "sobrecitos individuales" de Avecrem, la botella de "plástico reciclado" de Font Vella, todos necesitaban el concurso de la novedad.

Teniendo en cuenta que la publicidad es uno de los integrantes de los mecanismos psicológicos que regulan la alimentación (Menéndez García y Franco Díez, 2009: 319) y que la sociedad de consumo ha desarrollado de manera exacerbada los postulados del sanismo, nada tiene de extraño que el discurso publicitario sea a su vez el encargado de difundir las innovaciones en el campo de la industria alimentaria y sus efectos benéficos en la salud. En este sentido, puede observarse que la salud y la ciencia suelen acompañar a la novedad. De este modo viene a establecerse una correlación entre novedad, ciencia y salud (v. apdo. 4.2.4).

Por último, la novedad es, en ocasiones, el fundamento de la bondad (v. apdo. 4.1.1). Son numerosos los productos que recurren al hecho de ser nuevos para acrecentar su bondad o la justifican mediante la novedad: "Hasta el momento no 
habrás probado unos cereales como estos. Son los nuevos cereales integrales de Grano Vita. Distintos y con una calidad sin igual" (1990).

\subsection{Proto-razones}

En última instancia cabe incluir una categoría que podría denominarse proto-razones. Es decir, se trata de razones que no se han desarrollado lo suficiente (economía y ecología) o que para aparecer en el discurso necesitan el soporte de otra (belleza). Estos argumentos se caracterizan por tener una presencia porcentual mínima (los primeros) o por carecer de autonomía (el segundo). Las proto-razones son: la belleza, la economía y la ecología.

\subsubsection{La belleza}

A pesar de su omnipresencia en el discurso publicitario actual, la belleza no forma parte de las razones mayores, pues su aparición, como en el caso de la ciencia, depende de la presencia de la salud y la bondad. Es decir, la belleza siempre figura formando parte del dúo salud-belleza o del trío bondad-salud-belleza, lo que indica que la belleza es el resultado del consumo de productos que, debido a su bondad, inciden favorablemente en la salud y de ésta se deriva la consecución de un cuerpo sano y, por tanto, hermoso.

En la publicidad se establece una estrecha correlación entre estos tres conceptos, de forma tal que uno de ellos (belleza) es inseparable de los otros (bondad y salud). O al revés, la consumición de productos bondadosos y salutíferos conlleva la obtención de un cuerpo saludable, y un cuerpo sano es indefectiblemente bello. Y éste es el lugar común en el que se fundamentan todos los entimemas de la publicidad de alimentos moderna: la salud -entendida como delgadez, que se logra mediante el consumo de productos saludables- proporciona belleza. Salvo un caso de los años treinta (“Conserve la línea sin dejar de alimentarse”, carne líquida del Dr. Valdés García), la preocupación por la imagen corporal es de reciente aparición. Los anuncios que esgrimen esta razón de compra aparecen a partir de los años setenta, lo que ratificar la observación hecha por diversos analistas según la cual la expansión de la corpolatría coincide con el desarrollo del capitalismo (Juárez, 2007: 31; Ristovsky-Slijepcevic et al., 2010: 322). Es, por tanto, la implantación de la sociedad de consumo la que provoca que "el somatocentrismo se convierta en una de las psicosis del hombre moderno" (Rey, 2010: 148).

La belleza como argumento de venta aparece en los años setenta, se consolida en los ochenta y desde entonces presenta una trayectoria ascendente. Esta obsesiva preocupación por la belleza es una vuelta de tuerca de los postulados sanistas e higienistas. Ya no basta tener un cuerpo bien alimentado (sano) ni bien aseado (limpio). Tiene que ajustarse además al canon establecido (bello). La aspiración del hombre moderno se condensa en la siguiente consigna: "Bello (sano) por dentro y bello (limpio, hermoso) por fuera”. Y a este empeño de belleza contribuye la publicidad de alimentos. 
La belleza va indisolublemente unida a la salud (v. apdo. 4.1.4). Así puede verse en los siguientes anuncios: “Tab ¡sin azúcar! El refresco ideal para gente de buen ver. Porque ayuda a mantener tu figura” (1970), “¿Por qué acumular grasas que cansan y afean? La leche descremada Ram contiene proteínas, un complejo vitamínico adicionado y carece de las grasas que puedan suponer un peligro para la salud" (1980). En menor proporción, se combina con la bondad (v. apdo. 4.1.1): "Ahora ya no tienes que renunciar al sabor de una buena mayonesa para mantenerte en forma" (Ybarra light, 1980), "El queso para untar Bonsi es suave y cremoso, lo puedes combinar con todo y lo que es más importante, controlarás tu línea sin renunciar al sabor del mejor queso. Bonsi es un mundo a tu medida" (1990). La belleza se entiende ahora como la consecuencia, no del consumo de productos saludables-insípidos, sino de productos saludables-sabrosos. La belleza es, pues, el broche de oro de la solución a las paradojas alimentarias antes vistas.

\subsubsection{La economía}

El precio es una de las variables del marketing clásico y, también, está considerado uno de las razones de compra tradicionales de la publicidad. Sin embargo, a tenor de los resultados obtenidos, puede observarse que la razón económica es apenas significativa en el contexto de las razones encontradas. El precio no es un motivo de peso en la publicidad de alimentos española, ni siquiera en los arduos años cuarenta y cincuenta. Las pocas veces que aparece el precio lo hace repartido a lo largo del siglo de manera muy discreta ${ }^{3}$.

\subsubsection{La ecología}

La ecología, entendida como rechazo de todo producto cuya fabricación o componentes atenten contra la Tierra, apenas tiene representación entre las razones de compra halladas. Podría pensarse que es una razón moderna y por ello su porcentaje es casi nulo. Sin embargo, también se han encontrado razones recientes (el envase o la tradición) cuyos porcentajes son considerables. De los datos obtenidos cabe inferir que el argumento ecológico, en el campo de la alimentación, no ha alcanzado la frecuencia ni la intensidad que ha desarrollado en otros ámbitos. Dada su naturaleza, no es una razón autónoma, sino que, en los raros casos en los que aparece, sirve para fundamentar la bondad del producto: "Pizzas ecológicas. (BioLine, 2000)" o la "verdura ecológica" (2000).

3 En los años setenta se han encontrado varios anuncios en los que se utiliza el precio como razón de compra ("precios anticrisis"), de la misma manera que en la actualidad algunos anunciantes recurren al precio como argumento de venta. Como puede observarse, se trata de dos épocas de marcada crisis económica. 


\section{Conclusiones}

Como conclusión de cuanto se ha expuesto se obtienen tres conclusiones relativas a la trayectoria de las razones de compra, la prelación de dichas razones y el cambio de los hábitos alimentarios.

En cuanto a la trayectoria, se observa que unas razones de compra recorren todo el siglo y otras aparecen en el último tercio. Entre las primeras -las tradicionales- se encuentran la bondad, la salud, la variedad, la ciencia y la novedad. Todas ellas, a pesar de ser razones antiguas, muestran un incremento en las tres últimas décadas. También entre estas razones hay que ubicar la autenticidad, la economía y el estatus. Las razones de reciente aparición -las modernas- son: el envase, la facilidad, la tradición, la ecología y la belleza. Las nuevas y las viejas razones tienen un elemento común: las modernas hacen acto de presencia a partir de los años sesenta y justo en estos años algunas de las tradicionales viven un reverdecimiento.

Todo ello viene a indicar que la década de los sesenta marca una inflexión importante en la historia de la publicidad y del consumo españoles. En este decenio tiene lugar la consolidación económica iniciada años atrás, lo que supone el desembarco de numerosas agencias y empresas multinacionales; y el ingreso en la sociedad de consumo, que se afianza en los años siguientes. También en esta década, como consecuencia de lo anterior, se inicia el cambio de hábitos consumísticos, entre los que hay que situar los relativos a la alimentación.

En cuanto a la prelación, puede observarse que los valores a los que los españoles, en la actualidad, otorgan su favor de manera preferente son la bondad y la salud, que a fines del siglo XX constituyen, con la ciencia y la belleza, el cuarteto dominante. La medicalización de la sociedad, la divulgación del conocimiento científico, la visión ortoréxica de la alimentación y el deseo de poseer un cuerpo hermoso, todo ello determina que la belleza (como manifestación de la bondad del producto y de sus propiedades salutíferas, fundamentadas ambas en su carácter científico) se convierta en la razón de compra por excelencia.

La irrupción de empresas extranjeras y la proliferación de productos diferentes en el último tercio dan lugar al nacimiento de dos nuevas razones, que, respecto al cuarteto dominante, ocupan un segundo plano. Se trata del envase en el que se presenta el producto y de la facilidad con que se usa, prepara o consume. Al calor de ambas florecen dos viejos argumentos: la novedad, pues hay que encomiar lo flamante, lo moderno; y la variedad, que es la expresión del individualismo de la sociedad de consumo: cada consumidor debe tener su producto apropiado, de ahí la importancia de lo diverso, lo diferente.

Ya en un tercer plano, junto a la novedad figura -paradójicamente- la tradición como una suerte de nostalgia del pasado rural sepultado por la industrialización; y la autenticidad, que ahora se aplica a cualquier tipo de producto. Como antaño, el estatus convierte la alimentación en símbolo. Y la economía y la ecología apenas tienen importancia. 
En última instancia, a tenor de la trayectoria de las razones de compra y de la evolución de las preferencias de los consumidores, puede observarse que los hábitos alimentarios de los españoles se han transformado a lo largo del siglo XX. La elección de una u otra razón de compra para promocionar un producto depende de varios factores, uno de ellos es la mayor o menor "adhesión", para usar la expresión de Perelman, que despierte entre los consumidores, lo que viene a indicar que la publicidad está muy atenta a los cambios sociales. En este sentido, puede verse cómo, a raíz del desarrollo tecnológico, la evolución social y los cambios del imaginario colectivo, aparecen nuevas razones de compra o bien viejas razones reverdecen o modifican parcialmente su significado, como ha sucedido con "bueno", que, según unas u otras épocas, se ha ido asimilando a valores diversos.

Estos cambios indican, en términos generales, que España ha pasado de ser un país de un consumo limitado y homogéneo a comienzos del siglo XX a convertirse en un país de un consumo amplio y heterogéneo a comienzos del XXI. También manifiestan que la mayor transformación se ha producido a partir de los años sesenta, transformación que hay que situar en un contexto económico social determinado. Si Cussó y Garrabou hablan de “transición nutricional”, entendiendo por tal la convergencia alimentaria de España con los países de su entorno (2007), ahora, implantada la sociedad de consumo, puede hablarse de "segunda transición nutricional", según la cual la equiparación con dichos países no se lleva a cabo en el ámbito de los tres productos estudiados (cereales, patatas y legumbres), sino que se extiende a todos los productos, lo que supone una auténtica transformación de los hábitos alimentarios respecto a épocas anteriores.

Tabla I. Presencia absoluta (\%)

\begin{tabular}{|l|l|}
\hline Bondad & 27,66 \\
\hline Salud & 25,87 \\
\hline Ciencia & 9,45 \\
\hline Variedad & 7,69 \\
\hline Tradición & 6,21 \\
\hline Novedad & 4,97 \\
\hline Envase & 4,72 \\
\hline Autenticidad & 3,98 \\
\hline Belleza & 3,73 \\
\hline Estatus & 3,23 \\
\hline Economía & 0,99 \\
\hline Facilidad & 0,74 \\
\hline Ecología & 0,49 \\
\hline
\end{tabular}

Fuente: elaboración propia. 
Tabla II. Presencia en solitario (\%

\begin{tabular}{|l|l|}
\hline Estatus & 50,28 \\
\hline Envase & 36,64 \\
\hline Bondad & 33,10 \\
\hline Variedad & 32,45 \\
\hline Tradición & 29,60 \\
\hline Facilidad & 20,32 \\
\hline Autenticidad & 19,15 \\
\hline Salud & 15,33 \\
\hline Novedad & 6,66 \\
\hline Ciencia & 4,47 \\
\hline Belleza & 0,00 \\
\hline Ecología & 0,00 \\
\hline Economía & 0,00 \\
\hline
\end{tabular}

Fuente: elaboración propia.

Tabla III. Evolución cronológica

\begin{tabular}{|l|l|l|l|l|l|}
\hline & 1900 & 1925 & 1950 & 1975 & 2000 \\
\hline Autenticidad & & & & & \\
\hline Belleza & & & & & \\
\hline Bondad & & & & & \\
\hline Ciencia & & & & & \\
\hline Ecología & & & & & \\
\hline Economía & & & & & \\
\hline Estatus & & & & & \\
\hline Envase & & & & \\
\hline
\end{tabular}




\begin{tabular}{|l|l|l|l|l|l|}
\hline & 1900 & 1925 & 1950 & 1975 & 2000 \\
\hline Facilidad & & & & & \\
\hline Novedad & & & & & \\
\hline Salud & & & & & \\
\hline Tradición & & & & & \\
\hline Variedad & & & & & \\
\hline
\end{tabular}

Fuente: elaboración propia.

\section{Referencias bibliográficas}

Adam, J.M., Bonhomme, M. (2000): La argumentación publicitaria. Retórica y elogio de la persuasión. Madrid: Cátedra.

Arpe Muñoz, C. (2008): “Alimentos enriquecidos y fortificados”, Nutrición y Salud, vol. 3, pp. 35-54.

Capanaga, P. (2003): Salsa probiótica. La lengua de la publicidad alimentaria. Pórtico: Zaragoza.

Contreras Hernández, J., y Gracia Arnaiz, M. (2008): "Preferencias y consumos alimentarios. Entre el placer, la conveniencia y la salud”, Estudios Sociales de la Fundación “laCaixa”, vol. 24, pp. 153-191.

Cussó Segura, X., y Garrabou Segura, R. (2007): “La transición nutricional en la España contemporánea. Las valoraciones del consumo de pan, patata y legumbres (1850-2000)”, Investigaciones de Historia Económica, vol. 7, pp. 69-100.

Davo, M.C., y Álvarez Dardet, C. (2003): “El genoma y sus metáforas. ¿Detectives, héroes o profetas?”, Gaceta Sanitaria, vol. 17/1, pp. 59-65.

Díaz Méndez, C., y Gómez Benito, C. (2008): “Conclusiones. Problemáticas actuales en torno a la alimentación, el consumo y la salud", Estudios Sociales de la Fundación “laCaixa”, vol. 24, pp. 247-267.

Díaz Rojo, J.A. (2001-2002): "La belleza es salud. La medicalización lingüística de la publicidad de cosméticos", Contextos, vol. 37-40, pp. 109-121.

- (2003): “Lenguajes y reclamos de salud en la publicidad de alimentos”, Anàlisi, vol. 30, pp. 217-224.

Díaz Rojo, J.A., Morant i Marco, R., y Pixton, D.W. (2005): “El sanismo lingüístico. Recursos retóricos en la publicidad y etiquetado de alimentos”, Revista de Investigación Lingüística, vol. 8, pp. 35-51.

- (2006): El culto a la salud y la belleza. La retórica del bienestar. Madrid: Biblioteca Nueva. 
Fazio, M.E. (2008): "Pragmática y argumentación en el discurso publicitario: el caso de la campaña de SanCor Bio en Argentina”, Pensar la Publicidad, vol. II/ 2, pp. 15-36.

Gracia Arnaiz, M. (1997): La transformación de la cultura alimentaria. Cambios permanentes en un contexto urbano (Barcelona, 1960-1990). Madrid: Ministerio de Educación y Cultura.

- (2007): “Comer bien, comer mal. La medicalización del comportamiento alimentario”, Salud Pública de México, vol. 49/3, pp. 236-242.

Juárez, M. (2007): “Alimentos funcionales y publicidad”, Alimentos, Nutrición y Salud, vol. 14/2, pp. 31-32.

Krippendorff, K. (2004): Content analysis. A introduction to it methodology. Thousand Oaks (California): Sage.

Marín Murillo, F., Armentia Vizuete, J.I., y Ganzabal Learreta, M. (2010): "La publicidad en las revistas femeninas y masculinas: reflejo de los estereotipos de género”, Doxa Comunicación, vol. 10, pp. 35-56.

Martínez Álvarez, J.R. (2008): "Evolución de los hábitos alimentarios en España. Las nuevas tendencias, los nuevos alimentos y su relación con la salud”, Nutrición y Salud, vol. 3, pp. 7-33.

Menéndez García, R.A., y Franco Díez, FJ. (2009): “Publicidad y alimentación. Influencia de los anuncios gráficos en las pautas alimentarias”, Nutrición Hospitalaria, vol. 24/3, pp. 318-325.

Montero, M., Rodríguez, N., Rodríguez, J.; y Del Río, J. (2010b): Historia de la Publicidad y de las Relaciones Públicas en España. Vol. II: La edad de oro de la comunicación comercial. Desde 1960 hasta 2000. Sevilla-Zamora: Comunicación Social.

Murcia, M.A., Parras, P., Jiménez, A.M., Vera, A.M., Martínez-Tomé, M., y Ruggieri, S. (2008): “Polifenoles y flavonoides. Su importancia en la prevención de enfermedades", Nutrición y Salud, vol.3, pp. 121-142.

Oliva, J., González, L., Labeaga, J.M., y Álvarez Dardet, C. (2008): “Salud pública, economía y sociedad. El bueno, el feo y el malo", Gaceta Sanitaria, vol. 22/6, pp. 507-510.

Perelman, Ch., y Olbrechts-Tyteca, L. (1994): Tratado de la argumentación. La nueva retórica. Madrid: Gredos.

Rey, J. (2010): “Publicidad de productos de alimentación y de productos vigoréxicos. ¿Una cuestión de límite?”, Icono 14, vol. especial, pp. 143-169.

Ristovsky-Slijepcevic, S., Bell, K., Chapman, G.E., Beagan, B.L. (2010): “Beign 'thick’ indicates you are eating, you are healthy and you have an attractive body shape: Perspectives on fatness and food choice amongst Black and White men and women in Canada", The International Journal of Health Sociology, vol. 19/3 (Sociology of Food and Health), Adelaide, pp. 317-329 Disponible en: de http://hsr.e-contentmanagement.com/page/22/submit-papers. Consultado el 8 de junio de 2011.

Sánchez Guzmán, J.R. (1985): Introducción a la teoría de la publicidad. Madrid: Tecnos.

Vallone, F. (2009): “Pequeños grandes clientes. La publicidad de sucedáneos de la leche materna en dos revista argentinas entre 1977 y 2006”, Salud Colectiva, vol. 5/1, pp. 87-105. 